\title{
Fluorescence Detected Soft XAS and MCD - Applications to Bioinorganic Systems
}

\author{
S. P. Cramer ${ }^{a, b}$, G. Peng ${ }^{a}$, J. Christiansen ${ }^{a}$, J. Chen ${ }^{a}$, J. van Elp ${ }^{b}$, S.J. George ${ }^{a}$, and A.T. Young ${ }^{a, b}$ \\ Department of Applied Science, University of California, Davis, California 95616 \\ Energy \& Environment Division, Lawrence Berkeley National Lab, Berkeley, California 94720
}

Recent developments in synchrotron radiation sources and end stations make $x$-ray magnetic circular dichroism a feasible experiment for metalloproteins. This paper describes the experimental apparatus and some results that indicate the promise of the technique.

\section{INTRODUCTION}

Nitrogenase, hydrogenase, photosystem II, and other enzymes contain paramagnetic metal clusters [1]. It is important to understand the individual metal oxidation states and the magnetic couplings under various conditions. This article illustrates the potential of the paramagnetic XMCD technique for bioinorganic studies.

\section{XMCD INSTRUMENTATION}

A special factor for XMCD experiments on metalloproteins is that the samples are paramagnetic. For an $\mathrm{S}=1 / 2$ system, $\mathrm{B}(\mathrm{T}) / \mathrm{T}(\mathrm{K})$ needs to be greater than 2 to obtain $90 \%$ magnetization. Also, since the samples are dilute (100-1000 ppm),

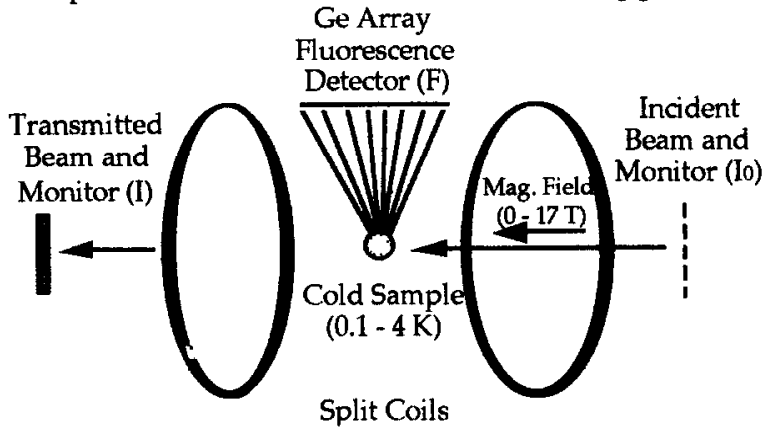

Figure 1. Schematic of XMCD experiment.

fluorescence detection is needed to extract the metal signal from the matrix background. Although the fluorescence detected excitation signal is not exactly the same as the true absorption coefficient, for many applications this problem can be overcome. A generic apparatus is shown in Figure 1.

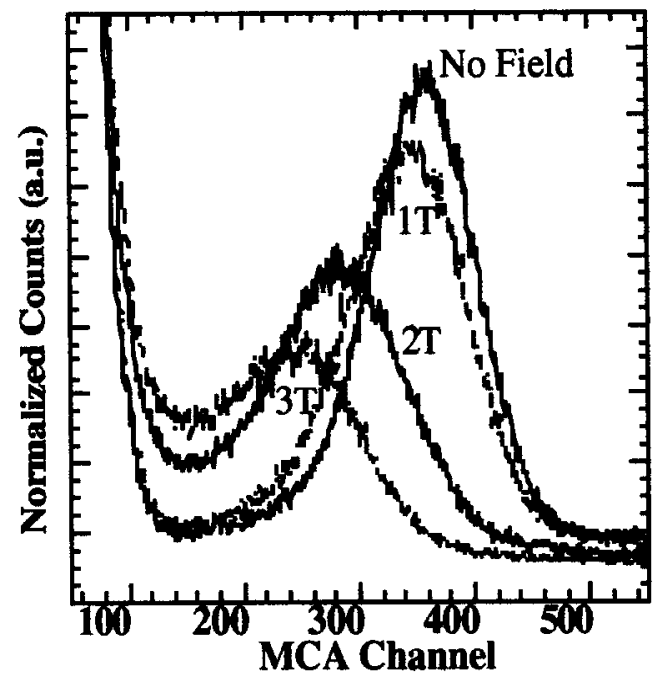

Figure 2. Magnetic Field Effects on Detector

The paramagnetic XMCD experiment was initially troubled with magnet quenches, inadequate thermal shielding, magnetic interference with the fluorescence detector (Fig. 2), and beamline instabilities. However, we have solved all 
of these problems, and recently XMCD has been an "almost" routine experiment.

With Janis Instruments, we have built 3 generations of XMCD instruments. The first used a pumped ${ }^{4} \mathrm{He}$ cryostat, and a $6 \mathrm{~T}$ magnet. Although the design worked, the high fields interfered with the detector performance (Figure 2). To allow $\mathrm{XMCD}$ at lower $B$ and $T$, we changed to a ${ }^{3} \mathrm{He}$ cryostat, which can achieve $\mathrm{T} \sim 0.3 \mathrm{~K}$. The shielding design is shown in Fig. 3. To confirm the actual sample temperature, we then recorded a magnetization curve for the $\mathrm{S}=1 / 2 \mathrm{Cu}^{2+}$ site in the 'blue' copper protein plastocyanin (Figures 4 and 5) [2].

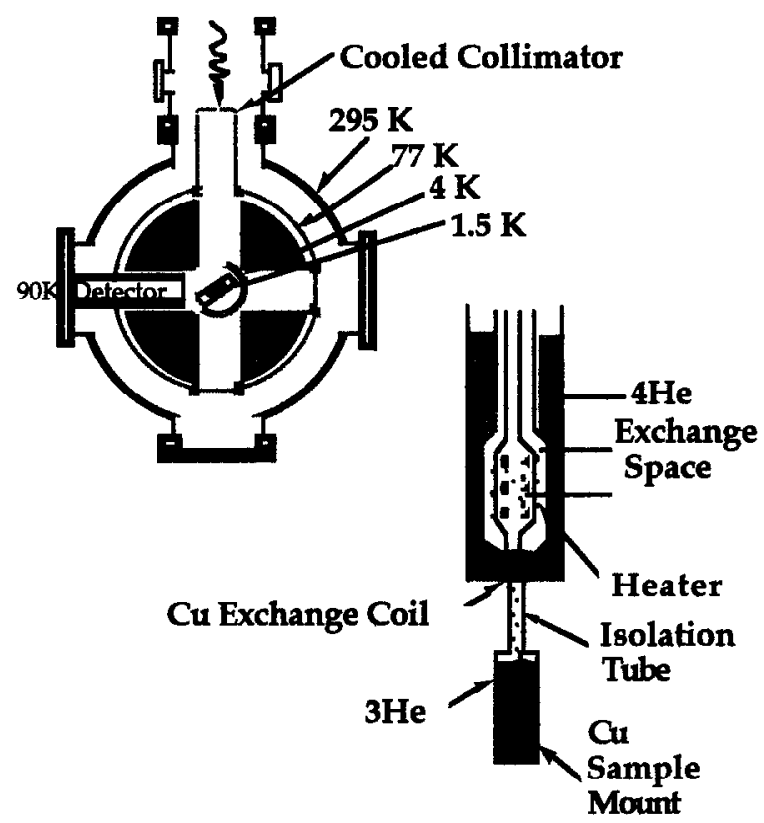

Figure 3. Shielding for the ${ }^{3} \mathrm{He}$ Cryostat

Our latest design uses a dilution refrigerator and a low inductance magnet. This allows us to reach $0.15 \mathrm{~K}$ or less, thus permitting XMCD measurements in very weak fields. The lower field will allow the fluorescence detector to move very close to the sample without losing resolution. The
XMCD of Cu Plastocyanin
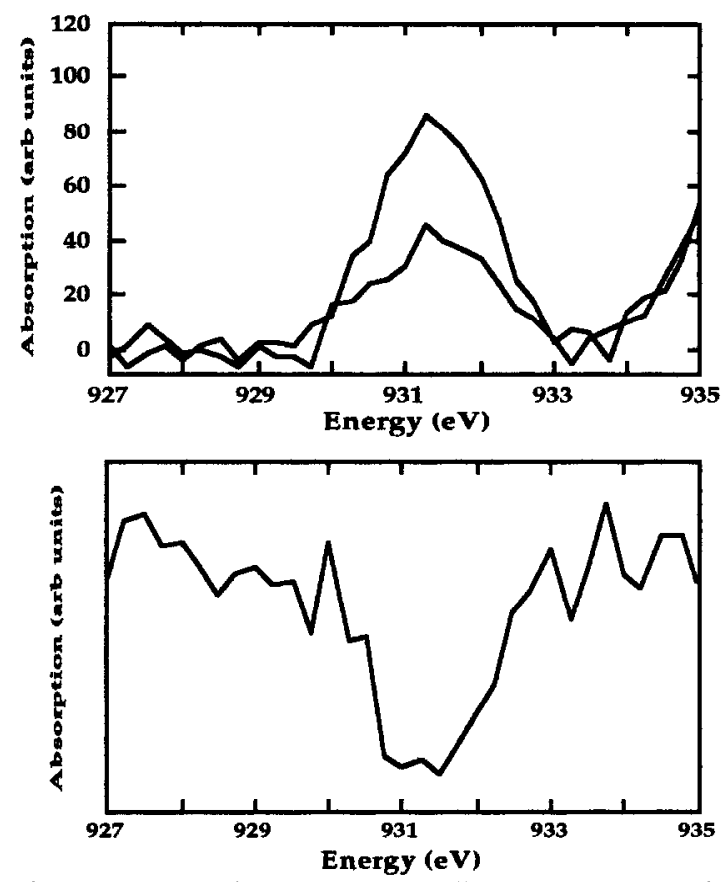

Figure 4. Plastocyanin Excitation and XMCD.

smaller fields and lower magnet inductance will allow us to change fields rapidly, comparing "spin-up" and "spin-down" spectra in seconds rather than hours.

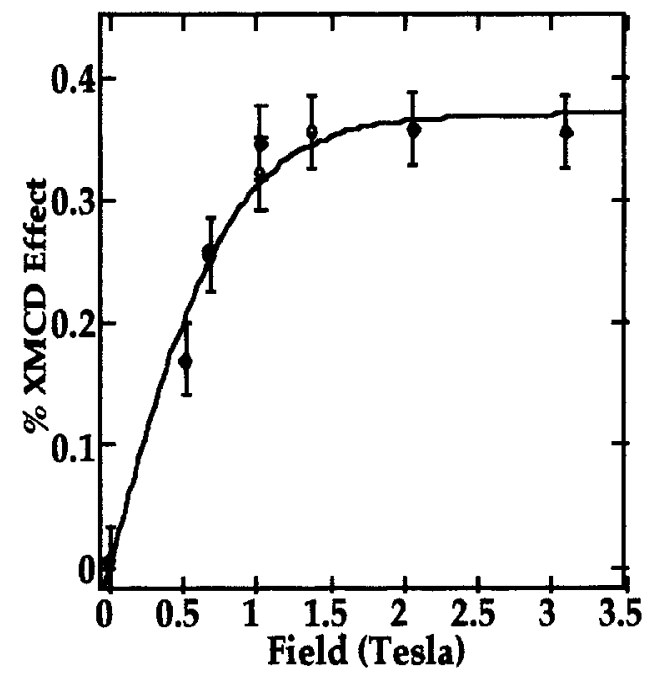

Figure 5. Plastocyanin Magnetization Curve 


\section{Metalloprotein Results}

Fe Rubredoxin. Our first XMCD experiment used the $\mathrm{Fe}$ (III) site in oxidized rubredoxin from P.yrococcus furiosus. (Figure 6) [3].

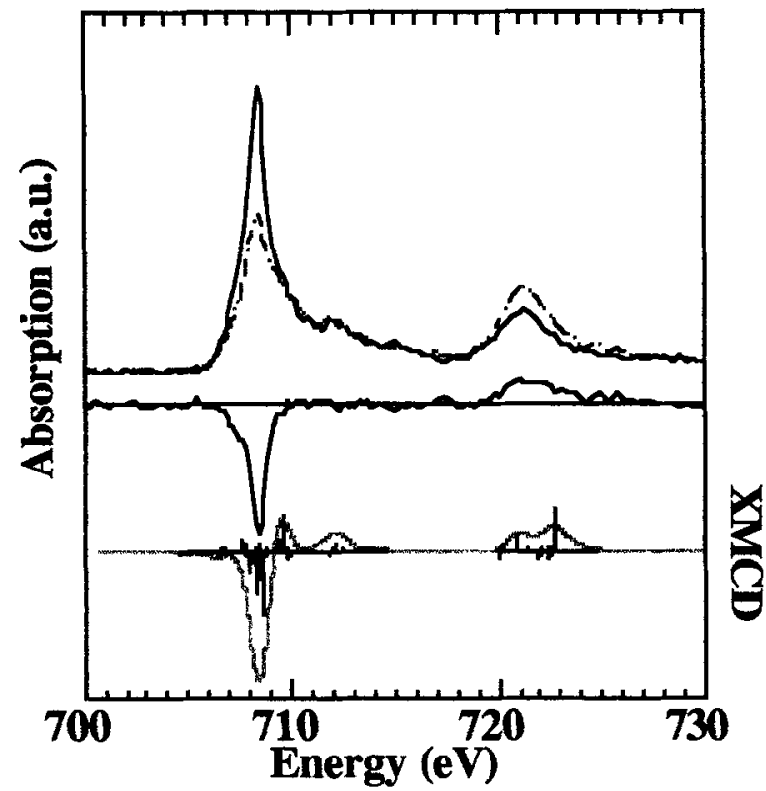

Figure 6. Rubredoxin $\mathrm{XMCD}$ and simulation.

Oxidized rubredoxin shows a strong $(\sim 30 \%) \mathrm{XMCD}$ at the Fe L/3-edge, with a broader signal of opposite sign at the $\mathrm{L}_{2-}$ edge. Apart from demonstration of strong $X M C D$, the rubredoxin work was important because of the experimental problems that were overcome. We have also examined and published [4] the L-edges XMCD for reduced Fe rubredoxin (Figure 6).

Plastocyanin. The blue copper protein plastocyanin contains an $\mathrm{S}=1 / 2, \mathrm{Cu}$ (II) site in its oxidized form. The lower magnetic moment means that this system is considerably more difficult to fully magnetize. Still, we observed a strong MCD effect (Figure 4), consistent with greater than $90 \%$ magnetization The $3 \mathrm{He}$ magnetization curve is in press [2].

Other Mononuclear Proteins. We have recorded Co L-edge data for the $\mathrm{Co}(\mathrm{II})$ substituted form of Pf rubredoxin (Fig. 7).
XMCD spectra were also obtained for myoglobin cyanide (Fig. 7). Here, a negative signal was found at both the $\mathrm{L}_{3}$ and $\mathrm{L}_{2}$ edges. Although we initially thought this must be an artifact, simulations show that this is the correct result for orbitally degenerate low-spin Fe(II). These mononuclear spectra form a base from which to examine more complicated mixed-metal and mixedvalence systems.

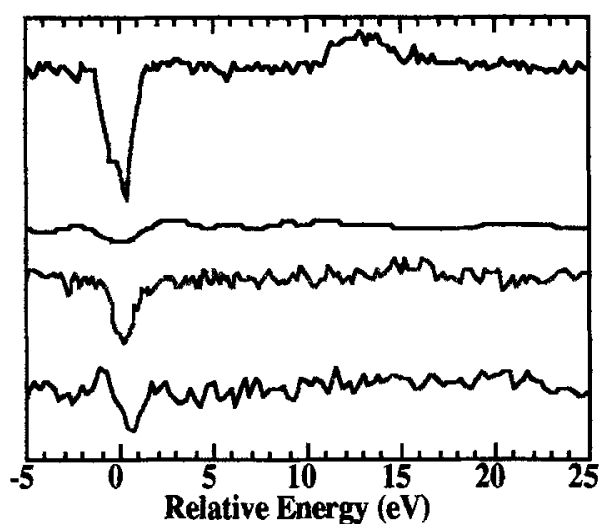

Figure 7. L-edge $\mathrm{XMCD}$ for (top to bottom): $\mathrm{Fe}(\mathrm{II})$ rubredoxin, $\mathrm{Fe}(\mathrm{III})$ myoglobin cyanide, $\mathrm{Co}(\mathrm{II})$ substituted rubredoxin, and a 'blue' Cu protein.

2-Fe Complexes. We recorded XMCD for $\left[\mathrm{Fe}_{2} \text { (II,III)(bpmp)(OPr) }{ }_{2}\right]^{2+}$, a weakly coupled mixed-valence complex (Fig. 8). In a

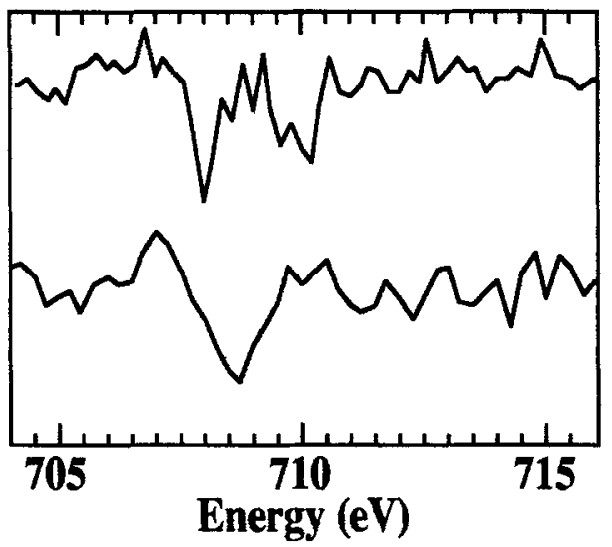

Figure 8. (Top) $\left[\mathrm{Fe}_{2}(\mathrm{II}, \mathrm{III})(\mathrm{bpmp})(\mathrm{OPr})_{2}\right]^{2+}$ and (bottom) $2 \mathrm{Fe}$ ferredoxin XMCD spectra. 
strong field, the Zeeman interaction is sufficient to overcome the $\left(J=\sim 10 \mathrm{~cm}^{-1}\right)$ exhange interaction, and signals with the same sign are found for both ferrous and ferric sites [5]. More surprising is the result seen for the strongly-coupled mixed-valence $\mathrm{Fe}(\mathrm{II})-\mathrm{Fe}$ (III) pair in 2Fe-2S ferredoxins. Preliminary data failed to show the expected bipolar XMCD signal. Work is in progress to obtain better signal to noise data at lower temperatures.

Nitrogenase. Nitrogenase is a good example of how XMCD selectivity for only the paramagnetic centers will allow investigation of complex metalloproteins. It has a molecular weight of 220,000 and contains 2 types of $\mathrm{Fe}-\mathrm{S}$ clusters - $8 \mathrm{Fe}-8 \mathrm{~S} \mathrm{P}$ clusters and MoFe7S9 M-centers (Fig. 9). In resting form the $\mathrm{P}$-clusters are diamagnetic,

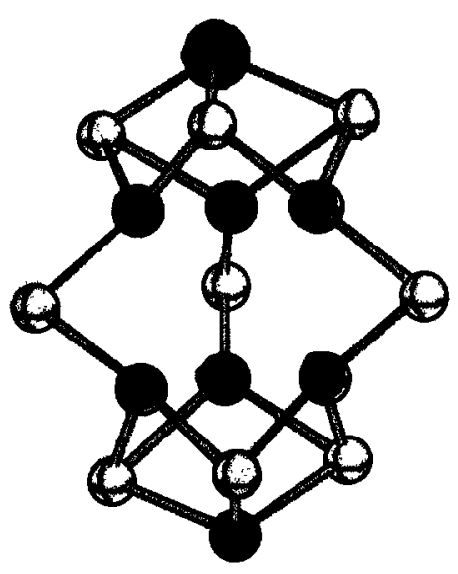

Figure 9. The nitrogenase $\mathrm{MoFe} 7$ cluster.

and the XMCD signal comes from the $S=3 / 2$ $M$ center, while in the thionin-oxidized form, the M-center becomes diamagnetic and the XMCD comes from the paramagnetic $(\mathrm{S}=3$ ?) P-clusters. Conventional L-edge spectra showed that the $\mathrm{Fe}$ absorption shifts to higher energy as Fe-S clusters become more oxidized (Figures 10-11). Thus, it might seem surprising that the nitrogenase $\mathrm{XMCD}$

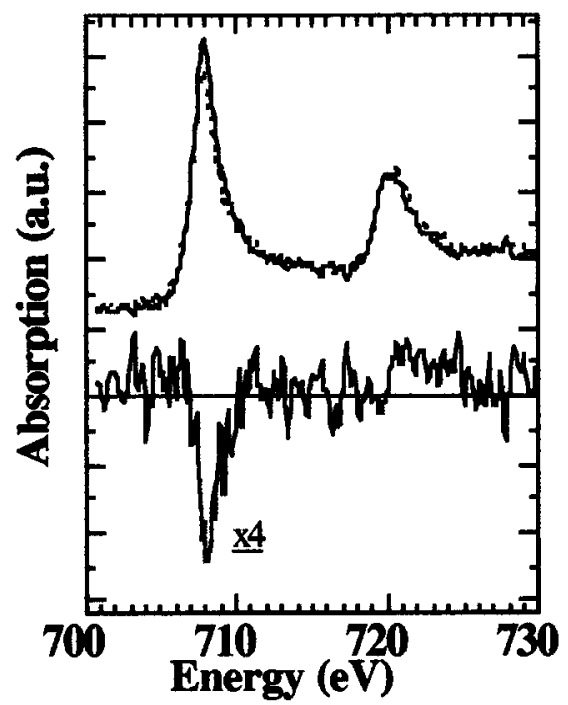

Figure 10 . Preliminary XMCD for the nitrogenase MoFe7 cluster.

shifts to lower energy upon thionin oxidation (Figure 11). However, this makes sense if the P-clusters are originally all $\mathrm{Fe}^{2+}$, and if thionin oxidation thus produces an XMCD signal from a cluster that is still mostly $\mathrm{Fe}^{2+}$.

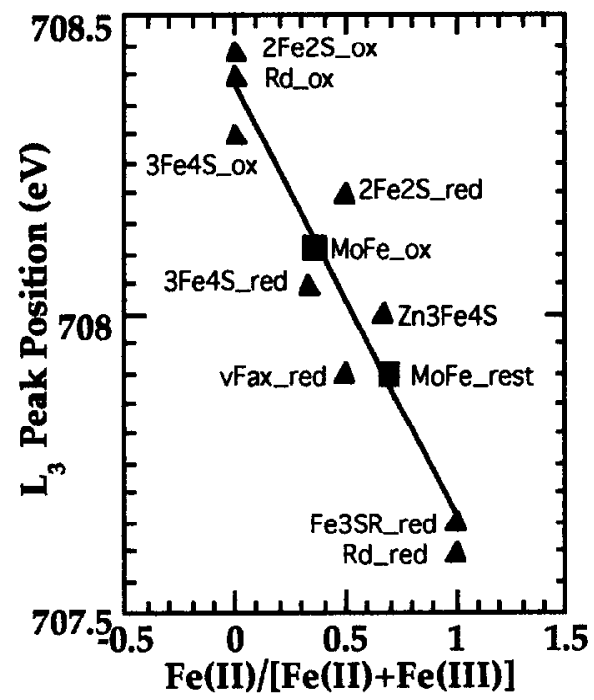

Figure 11. $\mathrm{L}_{3}$ position vs. Fe oxidation state. 


\section{CONCLUSIONS}

The experimental obstacles to conducting $\mathrm{XMCD}$ experiments on paramagnetic clusters in metalloproteins have been overcome. Additional work is needed to perfect the interpretation of fluorescence detected XMCD for clusters with complex magnetic spin Hamiltonians.

\section{References}

[1] "Principles of Bioinorganic Chemistry", S. J. Lippard and J. M. Berg, University Science, Mill Valley (1994)

[2] X-Ray Magnetic Circular Dichroism at Temperatures < $1 \mathrm{~K}$ : Demonstration with the Blue Copper Site in Plastocyanin,J. Christiansen, G. Peng, A. T. Young, L. B. LaCroix, E. I. Solomon, S. P. Cramer, Inorg. Chem. Acta. in press.

[3] "Soft X-ray Magnetic Circular Dichroism, a Probe for Studying Paramagnetic Bioinorganic Systems", J. van Elp, S. J. George, J. Chen, G. Peng, C. T. Chen, L. H. Tjeng, G. Meigs, H. J. Lin, Z. H. Zhou, M. W. W. Adams, B. G. Searle, and S. P. Cramer, Proc. Nat. Acad. Sci. USA. 90, 9664-9667 (1993).

[4] "Soft X-ray Magnetic Circular Dichroism on a Paramagnetic Bioinorganic System", J.van Elp, S.J. George, G. Peng, B.G. Searle, Z.H. Zhou, M. W. W. Adams, C.T. Chen, S.P. Cramer, SPIE Proc., San Diego (1993).

[5] C. Peng, J. van Elp, H. Jang, L. Que, W. Armstrong, and S. P. Cramer J. Am. Chem. Soc. 117, 2515-2519 (1995).

\section{ACKNOWLEDGEMENTS}

This work was supported by NSF DMB9107312, DIR-9105323, DIR-9317942, NIH GM- 44380 and by the DOE Office of Health and Environmental Research. SSRL is supported by DOE - Basic Encrgy Sciences. 